\title{
Anisotropic Decay Dynamics of Photoexcited Aligned Carbon Nanotube Bundles
}

\author{
Y. Hashimoto, ${ }^{1,2, \dagger}$ Y. Murakami, ${ }^{1,3}$ S. Maruyama,${ }^{3}$ and J. Kono ${ }^{1 *}$ \\ ${ }^{1}$ Department of Electrical and Computer Engineering, Rice University, Houston, Texas 77005, USA \\ ${ }^{2}$ Graduate School of Science and Technology and Venture Business Laboratory, Chiba University, Chiba 263-8522, Japan \\ ${ }^{3}$ Department of Mechanical Engineering, University of Tokyo 7-3-1 Hongo, Bunkyo-ku, Tokyo 113-8656, Japan
}

(Dated: May 9, 2018)

\begin{abstract}
We have performed polarization-dependent ultrafast pump-probe spectroscopy of a film of aligned single-walled carbon nanotube bundles. By taking into account imperfect nanotube alignment as well as anisotropic absorption cross sections, we quantitatively determined distinctly different photobleaching dynamics for polarizations parallel and perpendicular to the tube axis. For perpendicular polarization, we observe a slow (1.0-1.5 ps) relaxation process, previously unobserved in randomlyoriented nanotube bundles. We attribute this slower dynamics to the excitation and relaxation of surface plasmons in the radial direction of the nanotube bundles.
\end{abstract}

PACS numbers: 73.22.-f, 78.47.+p, 78.67.Ch

There is currently much interest in single-walled carbon nanotubes (SWNTs), both from scientific and technological points of view. Their unprecedentedly small diameters, combined with their unique molecular perfection, provide an ideal one-dimensional (1-D) playground for the exploration of new physical phenomena and novel applications. Their unique mechanical, electronic, optical, and magnetic properties are under intensive investigations world wide [1].

Various types of recent optical experiments, including ultrafast spectroscopy $2,3,4,4,5,6,67,8,9,10,11,12,13$, 14], have revealed some basic aspects of 1-D excitons with large binding energies in individualized semiconducting SWNTs. However, there has been very limited success in experimentally elucidating the intrinsically-anisotropic optical properties of SWNTs, despite the large number of detailed theoretical studies. This is primarily due to the lack of samples that contain aligned SWNTs of macroscopic sizes. Recently, vertically-aligned SWNT (VASWNT) films have been grown [15, 16, 17] using the alcohol chemical vapor deposition method [18, 19]. Although the bundled nature of SWNTs in these films preclude photoluminescence studies, they have allowed researchers to perform quantitative studies on anisotropic optical absorption cross sections of SWNTs 20]. Furthermore, an array of aligned SWNT bundles should be able to support unique collective plasma oscillations that cannot exist in individual SWNTs, which add new dimensions to the already rich optical properties of SWNTs.

Here we present results of polarization-dependent ultrafast pump-probe studies of a VA-SWNT film. We excited the first metallic subband and observed a clear difference in decay dynamics between parallel and perpendicular excitations. For polarization perpendicular to the tube axis, the decay of photoinduced transmission

*To whom correspondence should be addressed.; URL: http://www.ece.rice.edu/ kono Electronic address: kono@rice.edu change exhibited a slow (1.0-1.5 ps) component. We attribute this slow dynamics to the relaxation of surface plasmons excited in the perpendicular direction.

The VA-SWNT film used in this study was catalytically synthesized on both sides of a 0.5 -mm-thick optically-polished fused quartz substrate [15, 16, 17], using the alcohol chemical vapor deposition method 18, 19]. The characteristics of this type of VA-SWNT films have been described in great detail in a previous publication [17]. The average nanotube diameter, determined by high-resolution transmission electron microscopy, was $\sim 1.9 \mathrm{~nm}$, and most of SWNTs in the sample were bundles whose diameters ranged from 5 to $15 \mathrm{~nm}$. The thickness of the sample investigated in the present work was approximately $1 \mu \mathrm{m}$ in total. Linear optical properties of this sample, including its anisotropic absorption properties, have been fully characterized previously [20].

Polarization-dependent pump-probe experiments were performed using $\sim 150$-fs pulses from a mode-locked Ti:Sapphire laser operating at $80 \mathrm{MHz}$. The wavelength of both pump and probe beams was $800 \mathrm{~nm}$ (or $1.55 \mathrm{eV}$ ), which corresponded to the excitation energy of the first metallic subband of the sample 20]. The pump-probe intensity ratio was $\sim 10: 1$, and the signal-to-noise ratio in differential transmission $\Delta T / T$ was better than $10^{-5}$ using a Si photodiode with standard lock-in techniques. The pump fluence was kept small $\left(\mathrm{i} 640 \mathrm{~nJ} / \mathrm{cm}^{2}\right)$ in order to avoid high-density carrier effects $3,[4,[6]$, and the signal was proportional to the pump fluence. All measurements were performed at room temperature.

Figure 1(a) shows raw differential transmission data for $s$ - and $p$-polarizations taken at the incident angle $\theta$ $=60^{\circ}$, where $\theta$ is measured from the sample normal, as schematically shown in the inset of Fig. 1(a). The inset also indicates the direction of oscillation of the electric field of light for the two polarizations. The pump and probe polarizations were the same for the data shown in this figure. For both $s$ - and $p$-polarizations, the sign of $\Delta T / T$ is positive, i.e., photoinduced "bleaching" of absorption. The curve for the $s$-polarization is exactly the same as that taken at $\theta=0^{\circ}$ (shown later in the inset 


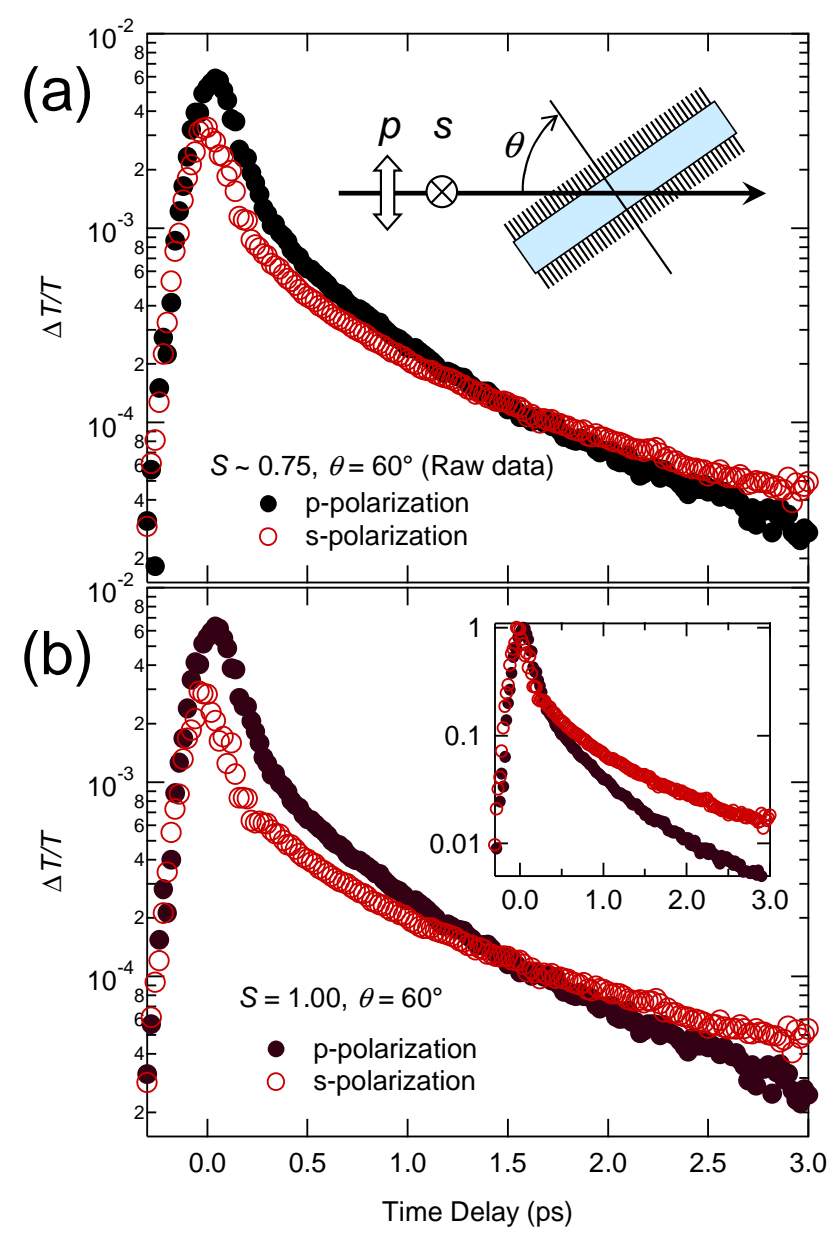

FIG. 1: Transient changes of transmission of the vertically aligned SWNT film, whose nematic order parameter $S \sim 0.75$, measured with $p$ - (filled circle) and $s$-polarizations (open circles) at photon energy $1.55 \mathrm{eV}$. (a) Raw data. The inset shows a schematic diagram of the experiment. (b) Expected data for the ideal alignment case $(S=1)$, calculated from (a) based on linear absorption cross sections. The inset shows the same data normalized to the maxima.

of Fig. 3) when scaled by a factor taking into account the difference in light path lengths, as expected from the sample's geometry.

In order to deduce quantitative information on anisotropic relaxation properties from the data in Fig. 1(a), we need to take into account the imperfect alignment of nanotubes in the sample. The nematic order parameter $S[21$ of the sample was previously determined to be 0.75 20]. In addition, the molar absorption cross sections (in $\mathrm{cm}^{2} /$ mole-C) of perfectly aligned SWNT bundles for light polarized parallel and perpendicular to the tube axis, $\sigma_{\|}$and $\sigma_{\perp}$, were previously determined, and their ratio $\sigma_{\perp} / \sigma_{\|}$is 0.21 at $1.55 \mathrm{eV}$ [20]. Through a full geometrical consideration, we calculated the overall optical absorption cross sections of a sample with order parameter $S$ for $s$ - and $p$-polarized light at incident angle $\theta, \epsilon_{p}(\theta, S)$ and $\epsilon_{s}(\theta, S)$, respectively; e.g., $\epsilon_{p}\left(60^{\circ}, 0.75\right)=0.647 \cdot \sigma_{\|}+0.353 \cdot \sigma_{\perp}$ and $\epsilon_{s}\left(60^{\circ}, 0.75\right)$ $=0.082 \cdot \sigma_{\|}+0.918 \cdot \sigma_{\perp}$ for the sample studied, whereas $\epsilon_{p}\left(60^{\circ}, 1\right)=0.75 \cdot \sigma_{\|}+0.25 \cdot \sigma_{\perp}$ and $\epsilon_{s}\left(60^{\circ}, 1\right)=\sigma_{\perp}$ for perfectly aligned $(S=1)$ bundles.

The linear transmittance of the sample for $p$-polarized (s-polarized) light at $\theta=60^{\circ}$ was $35 \%$ (65\%); i.e., $65 \%$ $(35 \%)$ of incident light is absorbed by the sample at this wavelength. This total absorption can be decomposed into the parallel and perpendicular components; the amounts of light energy absorbed by the two components are $I_{p, \|}=0.583 I_{0}$ and $I_{p, \perp}=0.067 I_{0}\left(I_{s, \|}=\right.$ $0.104 I_{0}$ and $\left.I_{s, \perp}=0.246 I_{0}\right)$, respectively, where $I_{0}$ is the incident light energy (in Joules). Since the overall temporal shape of the pump-probe signal did not vary with the pump pulse energy, we are in the linear regime. Thus, we assume that the observed signal can be decomposed into parallel and perpendicular components as

$$
[\Delta T(t) / T]_{i}^{S=0.75}=I_{i, \|} \cdot \beta_{\|}(t)+I_{i, \perp} \cdot \beta_{\perp}(t)
$$

where $i=s, p$ and $\beta_{\|}(t)$ and $\beta_{\perp}(t)$ are photo-induced differential transmission per unit pump energy absorbed by the sample (in $\mathrm{J}^{-1}$ ), corresponding to the $\sigma_{\|}$and $\sigma_{\perp}$ components of absorption, respectively. Figure 1(b) shows transient curves for the ideal case of $S=1.0$ (calculated using the $\beta_{\|}$and $\beta_{\perp}$ shown in Fig. 2 below), where the linear transmittances for $p$ - and $s$-polarizations would become $31 \%$ and $72 \%$, respectively. Slight but enhanced contrast between these two curves compared to the case of Fig. 1(a) is noticeable. The inset compares the transient curves normalized at their maxima, showing the existence of anisotropy in the magnitude of their curves especially in time delays $\& 1$ ps.

The different dynamics between parallel and perpendicular excitations can be most clearly seen when $\beta_{\|}(t)$ and $\beta_{\perp}(t)$ are separately plotted, as shown in Fig. 2 . They were obtained by solving the coupled equations, Eq. (1) for $i=s, p$, using the raw $[\Delta T(t) / T]_{i}^{S=0.75}$ data directly from Fig. 1(a). Here we clearly see that the perpendicular contribution has slower relaxation. It should be noted that $\beta_{\|}(t)$ and $\beta_{\perp}(t)$ have the unit of the inverse of absorbed energy density. Therefore, it is reasonable that they have nearly the same peak value at $t=0$, since the total number of $\pi$ electrons, which are the only electrons excitable at $1.55 \mathrm{eV}$, is the same.

The photoinduced transmission signal for excitation parallel to the SWNT bundles [i.e., $\beta_{\|}(t)$ ] shows faster decay; $90 \%$ of the decay occurs in the first $\sim 0.5 \mathrm{ps}$. This observation is similar to results in prior ultrafast optical studies of randomly-oriented bundles of SWNTs [22, 23, 24, 25, 26, 27, 28, 29]. Since parallel absorption dominates over perpendicular absorption in bundled SWNTs $\left(\sigma_{\|}: \sigma_{\perp} \approx 5: 1\right.$ at $\left.1.55 \mathrm{eV}[20]\right)$, it can be assumed that pump-probe signals in randomly-oriented bundles are dominated by carrier dynamics associated with parallel absorption. There are two dashed lines in Fig. 2, whose slopes correspond to exponential decay times of $140 \mathrm{fs}$ and $560 \mathrm{fs}$, respectively. This type of two-component decay has been invariably observed in the previous pump- 


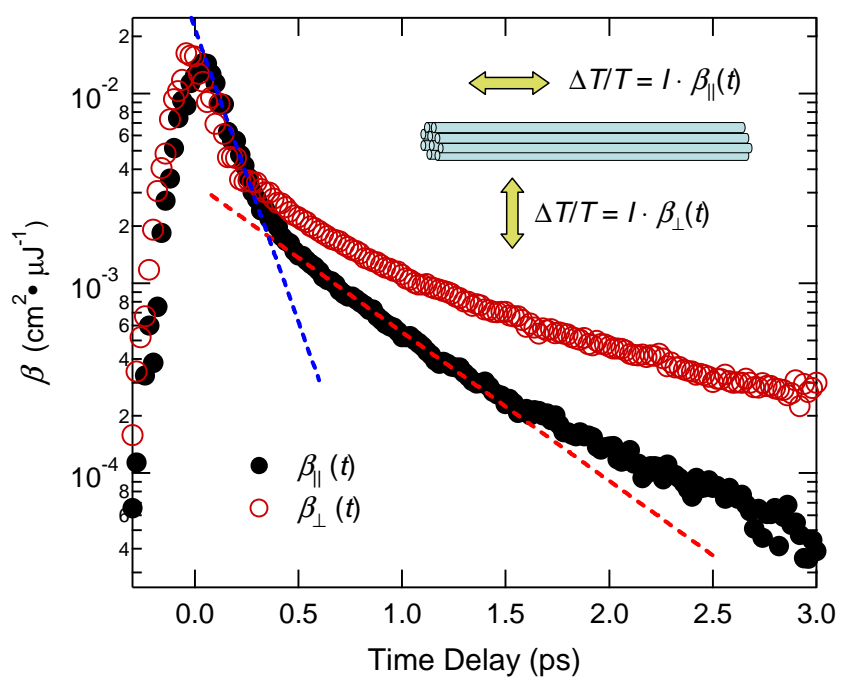

FIG. 2: Coefficients of the transient transmission caused by absorption of unit amount of pump energy by SWNTs, $\beta_{\|}(t)$ (filled circles) and $\beta_{\perp}(t)$ (open circles), for excitation polarization parallel and perpendicular to the SWNT axis, respectively. See Eq. (1) for the definitions. Inset shows a schematic of the corresponding polarization directions.

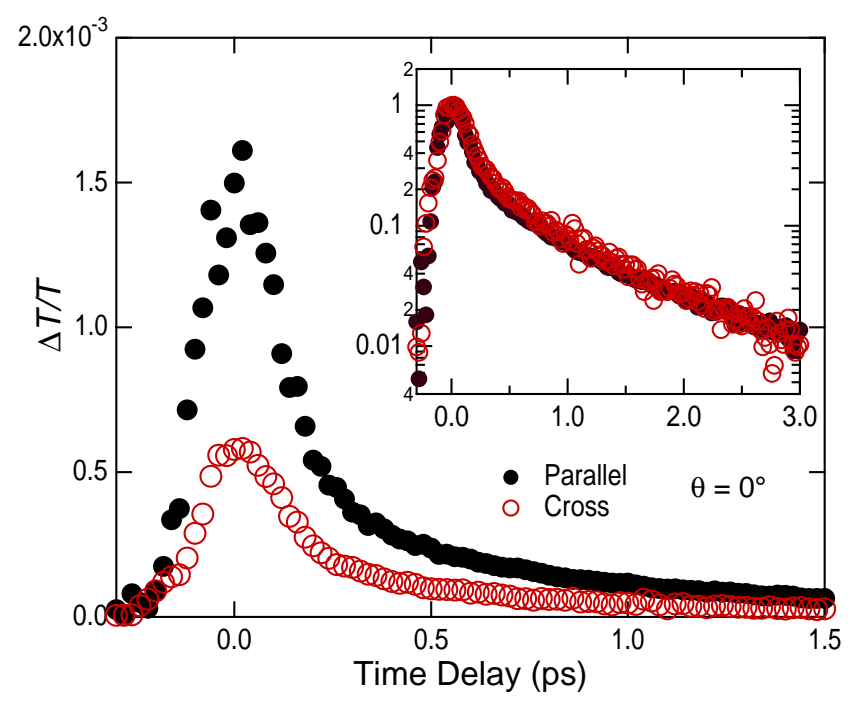

FIG. 3: Transient changes of transmission measured at normal incidence measured in "parallel" (filled circle) and "cross" (open circle) configurations. Inset shows the same curves normalized to the maxima.

probe studies and variously interpreted. The fast component can be due to electron-electron scattering 22], intraband relaxation [3, 11], or coherent "artifacts" [30]. The slower component is most likely due to carrier cooling due to electron-lattice interactions, but the gradual deviation of the $\beta_{\|}$trace from the 560 fs line means that the decay process between $\sim 500$ fs and 3 ps is not simple exponential, i.e., the decay becomes slower with time.

The slower dynamics observed only in $\beta_{\perp}$, i.e., per- pendicular excitation, has not been observed previously, and we regard this as an intrinsic property of the aligned SWNT bundles associated with charge dynamics in the radial direction. We have further evidence to confirm this point. Figure 3 shows pump-probe traces taken at normal incidence $(\theta=0)$, i.e., polarization $\perp$ tube axis. The two traces were recorded in parallel and cross configurations, respectively, in regards to the relative polarizations of the pump and probe beams. They differ in magnitude but completely agree with each other in time dependence, as shown in the inset, where they are normalized to their respective peak values and plotted in log scale, exhibiting the characteristic slower component. The amplitude ratio $\Delta T_{\|} / \Delta T_{\perp}$ is approximately 3.0 , which can be understood through a simple consideration of excitation of a cylindrical object. When charge dynamics is induced by linearly-polarized light oscillating in a direction perpendicular to the cylinder, the ideal changes in $T_{\|}$and $T_{\perp}$ due to the depolarization in the cylinder are given by

$$
\begin{array}{r}
\Delta T_{\|} \propto \int_{0}^{2 \pi} \cos ^{4} \phi d \phi \\
\Delta T_{\perp} \propto \int_{0}^{2 \pi} \cos ^{2} \phi \sin ^{2} \phi d \phi
\end{array}
$$

where $\phi$ is the azimuthal angle of the cylinder. The ratio of Eq. (2) to Eq. (3) is 3, which is in excellent agreement with the experimental result.

We propose that the slower dynamics we observed only for light polarization perpendicular to the tube axis $\left(\beta_{\perp}\right.$ in Fig. 2 and both traces in Fig. 3) arise from the excitation and relaxation of surface plasmons in the SWNT bundles. Since one third of SWNTs is metallic, and since one SWNT in a bundle has usually six neighbors adjacent to it [31], SWNT bundles can be regarded as conductive wires. It has been well established that optical excitation in conductive nanowires, whose diameter is small enough compared to the wavelength of incident light, is dominated by the dipolar surface plasmon excitation that is a collective excitation of electrons in their radial direction [32]. The surface plasmon mode in sufficiently long nanowires can only be excited by an electric field perpendicular to their axes because it is the only direction in which the excited electrons are spatially confined by the nanowire surfaces [33, 34]. The observed decay in the order of $1.0-1.5$ ps can be viewed as the characteristic time scale of the decay of the excited surface plasmons in the SWNT bundles. The reported relaxation times of surface plasmons in metallic nanoparticles range from sub-ps to a few ps and are believed to be determined by electron-phonon interactions $35,36,37,38,39]$.

However, there are at least two notable differences between SWNT bundles and metallic nanoparticles/nanowires. First, the diameter distribution of SWNT bundles (5-15 nm in the present sample [17]) is typically much broader than that of metallic nanoparticles [35]. Second, the sharp and strong extinction peaks in the case of metallic nanowires arise from plasmon reso- 
nance, in which the denominator of the extinction coefficient derived from Mie's theory becomes minimum when $\epsilon_{1}=-\epsilon_{m}$, especially when $\left|\epsilon_{2}\right|$ is small [32]. Here, $\epsilon_{1}$ and $\epsilon_{2}$ are the real and imaginary parts, respectively, of the dielectric function of the nanoparticle, and $\epsilon_{m}$ is the dielectric function of the surrounding medium. Recently, the values of $\epsilon_{1}$ and $\epsilon_{2}$ of SWNTs were reported for both parallel and perpendicular light polarizations in a wide spectral range [40], according to which $\epsilon_{1} \sim 0.5$ at $1.55 \mathrm{eV}$ for perpendicular polarization. When $\epsilon_{m}=1$ or larger and $0 ; \epsilon_{1} ; 1$, there is a local-field enhancement in the cylinder (i.e., the induced depolarization field has the same direction as the applied field) [32]. Thus, although the magnitude should be weaker than the resonant case, we may still excite a surface plasmon in the perpendicular direction.

In summary, we have studied the anisotropic decay dynamics of photo-excited aligned SWNT bundles. By exciting and probing the first metallic subband, we observed a clear difference in decay dynamics between parallel and perpendicular excitations. When the SWNT bundles are excited by perpendicularly-polarized light, the decay of photoinduced transmission change exhibited a slow (1.0-1.5 ps) component that was absent in the case of excitation by parallel-polarized light. Based on the fact that the SWNT bundles can be regarded as conductive nanowires, we attribute the observed slow component to the relaxation of excited surface plasmons in the perpendicular direction. In terms of use of the verticallyaligned bundled SWNT films as, e.g., ultrafast saturable absorbers [23, 41, 42, 43], the present results indicate that the combination of aligned SWNT bundles and incident pump light polarized parallel to the SWNT axes can provide the fastest turn-off speed, due to the absence of the slowly-decaying component.

This work was supported in part by the Robert A. Welch Foundation (through Grant No. C-1509) and the National Science Foundation (through Grant Nos. DMR-0134058 and DMR-0325474).

${ }^{\dagger}$ Present address: Imaging Science and Engineering Laboratory, Tokyo Institute of Technology, Yokohama, Kanagawa 226-8503, Japan
[1] See, e.g., M. J. O'Connell, ed., Carbon Nanotubes: Properties and Applications (CRC Press, Taylor \& Francis Group, Boca Raton, 2006).

[2] A. Hagen et al., Appl. Phys. A 78, 1137 (2004); Phys. Rev. Lett. 95, 197401 (2005).

[3] G. N. Ostojic et al., Phys. Rev. Lett. 92, 117402 (2004); ibid. 94, 097401 (2005).

[4] L. Huang, H. N. Pedrosa, and T. D. Krauss, Phys. Rev. Lett. 93, 017403 (2004); L. Huang and T. D. Krauss, Phys. Rev. Lett. 96, 057407 (2006).

[5] Y.-Z. Ma et al., J. Chem. Phys. 120, 3368 (2004); Phys. Rev. Lett. 94, 157402 (2005); J. Phys. Chem. B 109, 15671 (2005); Phys. Rev. B 74, 085402 (2006).

[6] F. Wang et al., Phys. Rev. Lett. 92, 177401 (2004); Phys. Rev. B 70, 241403(R) (2004).

[7] R. J. Ellingson et al., Phys. Rev. B 71, 115444 (2005).

[8] H. Hippler et al., Phys. Chem. Chem. Phys. 6, 2387 (2004).

[9] S. Reich et al., Phys. Rev. B 71, 033402 (2005).

[10] C.-X. Sheng et al., Phys. Rev. B 71, 125427 (2005).

[11] C. Manzoni et al., Phys. Rev. Lett. 94, 207401 (2005).

[12] S. G. Chou et al., Phys. Rev. B 72, 195415 (2005).

[13] R. M. Russo et al., Phys. Rev. B 74, 041405(R) (2006).

[14] H. Hirori et al., Phys. Rev. Lett. 97, 257401 (2006).

[15] Y. Murakami et al., Chem. Phys. Lett. 385, 298 (2004).

[16] S. Maruyama et al., Chem. Phys. Lett. 403, 320 (2005).

[17] Y. Murakami et al., Carbon 43, 2664 (2005).

[18] S. Maruyama et al., Chem. Phys. Lett. 360, 229 (2002).

[19] Y. Murakami et al., Chem. Phys. Lett. 374, 53 (2003).

[20] Y. Murakami et al., Phys. Rev. Lett. 94, 087402 (2005).

[21] R. G. Larson, The Structure and Rheology of Complex Fluids (Oxford University Press, New York, 1999).

[22] T. Hertel and G. Moos, Phys. Rev. Lett. 84, 5002 (2000);
Chem. Phys. Lett. 320, 359 (2000); T. Hertel, R. Fasel, and G. Moos, Appl. Phys. A 75, 449 (2002).

[23] Y.-C. Chen et al., Appl. Phys. Lett. 81, 975 (2002).

[24] M. Ichida et al., Physica B 323, 237 (2002).

[25] J. S. Lauret et al., Phys. Rev. Lett. 90, 057404 (2003); Phys. Rev. B 72, 113413 (2005).

[26] H. Han et al., Appl. Phys. Lett. 82, 1458 (2003).

[27] O. J. Korovyanko et al., Phys. Rev. Lett. 92, 017403 (2004).

[28] A. Maeda et al., Phys. Rev. Lett. 94, 047404 (2005); J. Phys. Soc. Jpn. 75, 043709 (2006).

[29] D. J. Styers-Barnett et al., J. Phys. Chem. A 109, 289 (2005).

[30] C.-X. Sheng and Z. V. Vardeny, Phys. Rev. Lett. 96, 019705 (2006); H. Okamoto et al., ibid. 96, 019706 (2006).

[31] A. Thess et al., Science 273, 483 (1996).

[32] U. Kreibig and M. Vollmer, Optical Properties of Metal Clusters (Springer, Berlin, 1995).

[33] G. Schider et al., J. Appl.Phys. 90, 3825 (2001).

[34] M. Barbic et al., J. Appl.Phys. 91, 9341 (2002).

[35] S. Link and M. A. El-Sayed, J. Phys. Chem. B 103, 8410 (1999).

[36] C. Voisin et al., J. Phys. Chem. B 105, 2264 (2001).

[37] T. Tokizaki et al., Appl. Phys. Lett. 65, 941 (1994).

[38] J. H. Hodak, I. Martini, and G. V. Hartland, J. Phys. Chem. B 102, 6958 (1998).

[39] H. Inouye et al., Phys. Rev. B 57, 11334 (1998).

[40] J. A. Fagan et al., unpublished.

[41] S. Tatsuura et al., Adv. Mater. 15, 534 (2003).

[42] S. Yamashita et al., Optics Lett. 29, 1581 (2004).

[43] Y. Sakakibara et al., Jpn. J. Appl. Phys. 44, 1621 (2005). 